\title{
Geochemical and Mineralogical Characteristics of the Mayouom Kaolin Deposit, West Cameroon
}

\author{
B. Tassongwa ${ }^{1,2}$, C. Nkoumbou ${ }^{2}$, D. Njoya ${ }^{3}$, A. Njoya ${ }^{4,5}$, J. L. Tchop ${ }^{2,6}$, J. Yvon ${ }^{7} \&$ D. Njopwouo ${ }^{3}$ \\ ${ }^{1}$ Department of Earth Sciences, Faculty of Sciences, University of Dschang, Dschang, Cameroon \\ ${ }^{2}$ Department of Earth Sciences, Faculty of Sciences, University of Yaounde I, Yaoundé, Cameroon \\ ${ }^{3}$ Department of Inorganic Chemistry, Faculty of Sciences, University of Yaounde I, Yaoundé, Cameroon \\ ${ }^{4}$ Institut des Beaux Arts de Foumban, Université de Dschang, BP 31 Foumban, Dschang, Cameroon \\ ${ }^{5}$ Mission de Promotion des Matériaux Locaux (MIPROMALO), B.P. 2396 Yaoundé, Cameroon \\ ${ }^{6}$ Institut de Recherche Géologique et Minière (IRGM/ARGV), Buea, Cameroon \\ ${ }^{7}$ Université de Lorraine, Laboratoire Environnement et Minéralurgie, UMR 7569, INPL-CNRS, 15 Avenue du \\ Charmois, BP 40 F-54501 Vandoeuvre, lès-Nancy, Nancy, France \\ Correspondence: B. Tassongwa, Department of Earth Sciences, Faculty of Sciences, University of Dschang, \\ Dschang, Cameroon. Tel: 237-7769-3065. E-mail: btassongwa2004@yahoo.fr
}

Received: October 1, 2013 Accepted: October 23, 2013 Online Published: January 23, 2014

doi:10.5539/esr.v3n1p94 URL: http://dx.doi.org/10.5539/esr.v3n1p94

\begin{abstract}
Geological, mineralogical and geochemical studies were carried out on clay materials of the same pit from Mayouom kaolin deposit located within a mylonitic shear zone about $30 \mathrm{~km}$ north of Foumban town (western Cameroon), in order to define their characteristics, the ore genesis and its economic interest. Seven samples were studied using different techniques: description of the pit, optical microscopy, microprobe analysis, and bulk chemical analyses. Two different facies (sandy kaolin and sand-poor kaolin) were observed in the field. Microscopical observations show that the mylonitic schistosity is well conserved in sandy clays, while sand-poor clays reveal the transformation of muscovite/illite into kaolinite. Quantitative and qualitative mineralogical investigations reveal kaolinite as predominant mineral, associated to quartz + illite/muscovite + anatase \pm hematite $\pm \mathrm{Ba}$, Sr-hydroxyapatite. Kaolinite (54\% and $81-84 \%$ respectively) mineral presents homogeneous shape and a good crystallinity. REE pattern show a Ce negative anomaly marking a reductive milieu of alteration.

All these results point out a hydrothermal alteration of feldspars and mica-rich rocks as petrogenetic origin of kaolins. Sandy kaolin comes from mylonites basement while sand-poor kaolin is related to the alteration of magmatic intrusive shape veins.

Due to its high kaolinite content (up to 85\%) and the low iron mineral content (less than 1.5\%), Mayouom kaolin is a suitable raw material for white burning industrial clays.
\end{abstract}

Keywords: Cameroon, Mayouom, kaolin, hydrothermal alteration

\section{Introduction}

For more than a decade, the clay market, and especially that of kaolin has been seriously affected by the overproduction in Europe and USA, the emergence of high quality kaolin source in Brazil and Australia, and the increasing supply of low-grade materials (Gougazeh \& Buhl, 2010; Nyakairu et al., 2001). Africa and especially Cameroon is one of the most consumers of clay derived products from China and European countries. This constitutes a lot of expenditure for the country. Numerous clay deposits are being discovered in the country, such as: a friable halloysite deposit from Mount Bamenda at Santa, Bali, Bambili and Sabga areas (Ossah, 1975), more than 15 m thick kaolinitic clays at Lembo and Bana, (Wouatong et al., 1996; Wouatong, 1997; Pialy et al., 2008, 2009), the Bomkoul fire bricks clay deposit (Njopwouo,1984; Njopwouo et al., 1998; Ngon et al., 2012); but the valorisation of clays material for industrial applications (raw materials for ceramic, paint, paper...) often needs the mastering on the processes of the clay deposit genesis (Murray \& Keller, 1993) and the mineral quality (Bloodworth et al., 1993; Mitchell, 1994; Merabet \& Belkacemi, 2003; Martin, 2005). 
The Mayouom kaolin deposit is found within the north-eastern Foumban mylonitic shear zone (Njonfang et al., 1998) composed of mylonites and late volcanic rocks. The presence of both supergene (meteoritic) and hypogene (hydrothermal) clays is common in such geological environment (Cravero et al., 2010). Njoya et al. (2006) demonstrated that the deposit was originated from hydrothermal alteration. This paper presents details mineralogical and geochemical characteristics of a particular outcrop and pit (MYII) in the deposit, pointing out the possible vertical variation of the materials.

\section{Materials and Methods}

Seven samples were collected on a single outcrop by digging and drilling using an eight meters manual auger. This outcrop was chosen in order to study both sandy and sand-poor material on a single vertical section. At the lab, the petrography, geochemistry and mineralogy were studied by polarized-light microscopy of thin sections, microprobe analysis and bulk chemical analyses. Petrography was performed on hardened thin slides of undisturbed samples.

Chemical analyses were performed at CRPG (Nancy, France) by emission spectrometry using inductively coupled plasma and atomic emission source (ICP-AES) for major elements, and mass spectrometry (ICP-MS) for trace elements and rare earths, after fusion with $\mathrm{LiBO}_{2}$ and dissolution in $\mathrm{HNO}_{3}$.

Mineral compositions were determined with an automated CAMECA SX100 electron microprobe (EMPA) at UHP. Operating conditions for silicates and oxides were $15 \mathrm{kV}$ acceleration voltages and $10 \mathrm{nA}$ probe current. The electron beam was enlarged at $5 \times 4 \mu \mathrm{m}$ and counting time was $30 \mathrm{~s}$ for each element $(15 \mathrm{~s}$ for Fe, $10 \mathrm{~s}$ for $\mathrm{Na}, \mathrm{K})$. Natural and synthetic oxides were used as standards, and the raw data were corrected on line for drift, dead time, and background, using X-PHI programs provided by CAMECA.

The quantitative mineralogical composition was calculated taking into account the results of qualitative mineralogy from microscopy observation, microprobe analysis and previous works. The calculations were done using the geochemical data and the formula T(a) = $\sum$ MixEl(Mi) (Yvon et al., 1982); where:

$\mathrm{T}(\mathrm{a})$ : wt.\% of element "a" in the sample;

Mi: wt.\% of the mineral "i" in the sample;

El(Mi): wt.\% of the element "a" in a mineral "i".

Therefore, the reconstitution scheme is as follow:

$-\mathrm{K}_{2} \mathrm{O}$ is used for muscovite (or illite) $\mathrm{KAlSi}_{3} \mathrm{O}_{10}(\mathrm{OH})_{2}$ and the wt $\%$ of $\mathrm{SiO}_{2}$ and $\mathrm{Al}_{2} \mathrm{O}_{3}$ are deduced according to their percentage in the mineral.

- The remaining $\mathrm{Al}_{2} \mathrm{O}_{3}$ is totally used for the calculation of Kaolinite $\left(\mathrm{Si}_{2} \mathrm{Al}_{2} \mathrm{O}_{5}(\mathrm{OH})_{4}\right)$ and the consumed $\mathrm{SiO}_{2}$ is deduced.

- After muscovite and kaolinite, the remaining $\mathrm{SiO}_{2}$ is considered as quartz;

- Ba, Sr-hydroxyapatite $(\mathrm{Ba}, \mathrm{Sr})_{5}\left(\mathrm{PO}_{4}\right)_{3}(\mathrm{OH})$ is calculated with $\mathrm{P}_{2} \mathrm{O}_{5}$;

$-\mathrm{TiO}_{2}$ is used for anatase $\mathrm{TiO}_{2}$ (detected by XRD);

$-\mathrm{Fe}_{2} \mathrm{O}_{3}$ is used for hematite.

\section{Results}

\subsection{Field Description}

The Mayouom area belongs to the Foumban shear zone where a mylonitic band extends in neoproterozoïc basement (500-660 Ma age), intrusive magmatic rocks (less than $40 \mathrm{Ma}$ age) and lodes (Njonfang, 1998). The deposit covers an area of about $500 \times 2000 \mathrm{~m}$ on the north western flank of the Mbatkom clift. It lies between 800 and $900 \mathrm{~m}$ altitude. The index of clay is essentially the dark grey to ash grey colour of soil (Njoya, 2006). During mining prospection of the deposit, more than seventy drills were done using a hand auger, with the maximum of $13 \mathrm{~m}$ deep, but the basement rock was not found. At MYII site (Figure 1) concerned with this paper $\left(5^{\circ} 51^{\prime} 25^{\prime \prime} \mathrm{N}, 10^{\circ} 59^{\prime} 20^{\prime \prime} \mathrm{E}\right)$, at $8 \mathrm{~m}$ deep, the clay was still very soft, with no sand traces as indication of basement rock, showing that the thickness of the deposit is greater.

On the outcrop, the MYII profile (Figure 2) presents a brownish grey colour. From the surface downwards, one can observe:

12 to $15 \mathrm{~cm}$ thick organo-mineral horizon, dark grey, covered with grass and bushes of which the roots cut the kaolin at $2 \mathrm{~m}$ deep. 
A red lateritic level, speckled with yellow and grey, with a thickness of 1.1 to $1.3 \mathrm{~m}$. This level is structurally and lithologically discordant with the kaolin it covers.

The kaolinic level made up of sandy and sand poor clays. The conserved mylonitic foliation is observable in sandy clays with an attitude N015E65 ${ }^{\circ} \mathrm{ESE}$.

Table 1 presents the characteristics of the samples collected from 2 to $8 \mathrm{~m}$ depth.

\subsection{Petrography}

Under the optical microscope, thin sections show fine texture with abundant matrix (70-85\%), with diverse colours (dark brown, dark grey, brownish grey). Sample MY22S presents the conserved mylonitic foliation (Figure 3a). Almost all kaolinite crystals are aligned following the inherited schistosity. Quartz crystal show "rolling extinction" (moving shadow) that characterise the mylonites of the region. MY22G and other sand poor samples are made up of more than $50 \%$ kaolinite, muscovite and dark matrix made up of oxides (Figure $3 b$ ). The vestiges of muscovite/illite in the process of kaolinization are observed all over the sections. Samples MY26, MY27 and MY28 present the same mineralogical composition but their texture is disturbed by the sampling technique (hand auger).

In addition to those minerals (Kaolinite + Quartz \pm Muscovite \pm oxides), X Rays and Infrared analysis have reveal $\mathrm{Ba}$, Sr-hydroxyapatite in clay materials from MYII pit, and that opaque minerals are hematite and anatase.

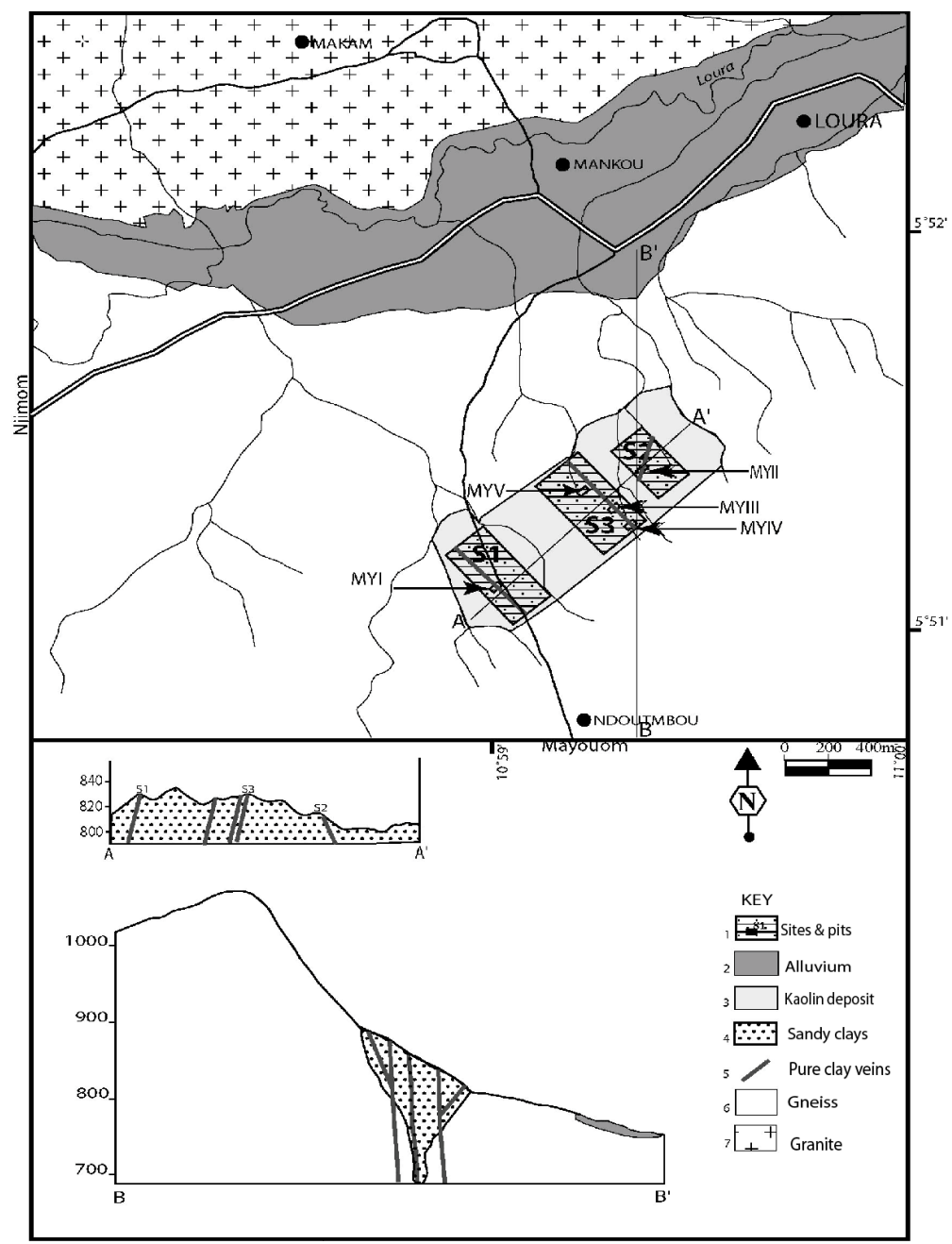

Figure 1. Geological map of the deposit and some geological sections (Njoya, 2007; Njoya et al., 2006) 
Table1. Physical characteristics of samples studied

\begin{tabular}{lccccccc}
\hline Samples & MY22S & MY22G & MY24 & MY25 & MY26 & MY27 & MY28 \\
\hline depth(m) & 2.80 & 2.80 & $3-4$ & $4-5$ & $5-6$ & $6-7$ & $7-8$ \\
Sight colour & Light brown & Light brown & Light brown & Greyish white & Greyish white & White & white \\
Texture & sandy & Clayey & Clayey & Clayey & Clayey & Clayey & Clayey \\
\hline
\end{tabular}
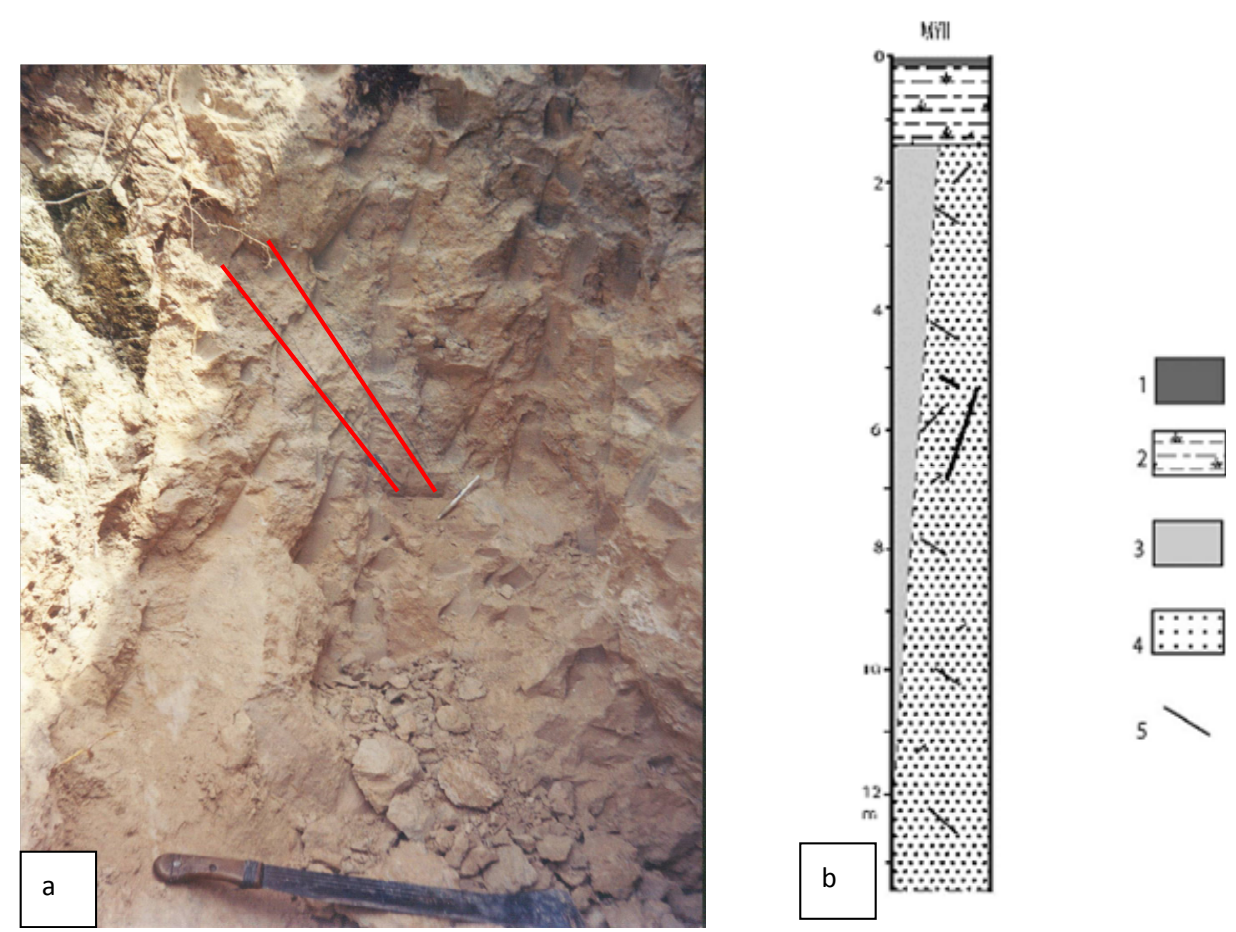

Figure 2. a) Pit MYII in the Mayouom clay deposit; b) Representative vertical cross section showing the two kaolin facies. 1: grey organo-clayey level; 2: lateritic soil; 3 : sand-poor vein clay; 4: sandy clay; 5 : quartz-feldspar veins
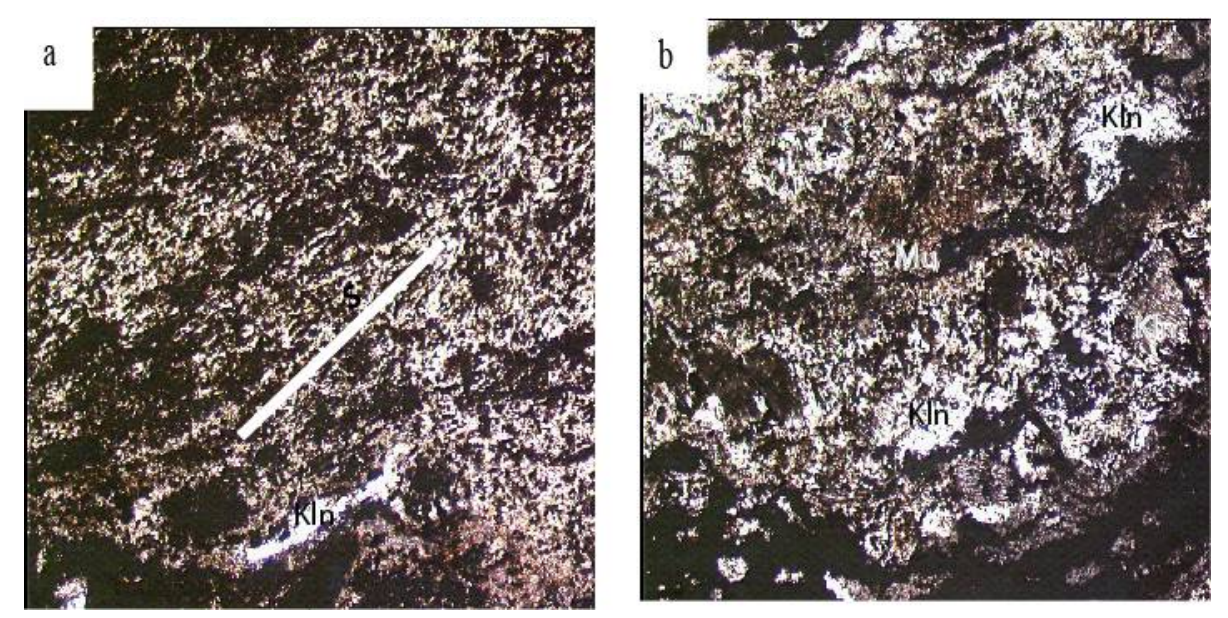

Figure 3. a) Sample MY22S in which the inherited mylonitic texture and schistosity (S) are well observed; b) MY22G with well formed crystals of Kaolinite (Kln) and muscovite (Mu)

\subsection{Mineralogy}

34 microprobe analyses were done on 4 sand poor samples (MY22G, MY24, MY26 and MY28). These analyses concerned kaolinite (Table 2), muscovite/illite and anatase (Table 3). Structural formulas were calculated with 
11 oxygens for kaolinite and muscovite, 2 oxygens for anatase $\left(\mathrm{TiO}_{2}\right)$. From these calculations it comes that kaolinite is essentially pure (up to $85 \%$ ); muscovite is made up of pure muscovite $\left(\mathrm{KAl}_{2}\left[\mathrm{Si}_{3} \mathrm{AlO}_{10}(\mathrm{OH}, \mathrm{F})_{2}\right]\right)$, illite $\left(\mathrm{K}_{\mathrm{x}} \mathrm{Al}_{2}\left[\mathrm{Si}_{4-\mathrm{x}} \mathrm{Al}_{\mathrm{x}} \mathrm{O}_{10}\right](\mathrm{OH})_{2}\right)$ and phengite $\left(\mathrm{K}_{2}(\mathrm{Fe}, \mathrm{Mg}) \mathrm{Al}_{3}\left[\mathrm{Si}_{7} \mathrm{Al} \mathrm{O}_{20}(\mathrm{OH}, \mathrm{F})_{4}\right]\right)$; anatase is pure. They are very few impurities of different nature. The most considerable impurity is FeOt; with the presence of anatase, this oxide explains the presence of opaque minerals in the microscope. Sr is totally absent while $\mathrm{Na}$ and $\mathrm{Ca}$ are fewly represented.

\subsection{Chemical Composition}

Major element (Table 4) and trace element (Table 5) compositions of studied materials show distinction between sandy and sand-poor clays. In sandy kaolin (MY22S), $\mathrm{SiO}_{2}, \mathrm{Al}_{2} \mathrm{O}_{3}$ and LOI content are $65.86 \%, 22.60 \%$ and $9.43 \%$ respectively, and a high chemical index of alteration (CIA) $\mathrm{SiO}_{2} / \mathrm{Al}_{2} \mathrm{O}_{3}$ (2.91). These chemical characteristics correlate with high quartz content. In the other hand, sand-poor kaolins contain lower $\mathrm{SiO}_{2}$ (43-46\%), higher $\mathrm{Al}_{2} \mathrm{O}_{3}$ (up to 35.47\%) and low CIA (1.22-1.34) due to higher kaolinite content and higher $\mathrm{TiO}_{2}(4 \%)$ due to the presence of anatase. The CIA increases with quartz content, from theorical pure kaolinite (1.18) to MY22S, the sandy sample (2.91).

In the two facies (sandy and sand-poor), iron content is very low (less than 1.2\%), inducing white or whitish colour of all materials. The small quantities of $\mathrm{K}_{2} \mathrm{O}$ related to illite/micas are present in all studied samples. The relatively low content of $\mathrm{TiO}_{2}, \mathrm{Fe}_{2} \mathrm{O}_{3}, \mathrm{MgO}, \mathrm{K}_{2} \mathrm{O}, \mathrm{P}_{2} \mathrm{O}_{5}$ oxides in sandy kaolins compared to sand-poor kaolins is apparent. The $\mathrm{MnO}, \mathrm{CaO}$ and $\mathrm{Na}_{2} \mathrm{O}$ are completely lost in both types of facies.

As a function of depth, the variation interval of major elements is narrow (Figure 4). Some elements are decreasing $\left(\mathrm{Fe}_{2} \mathrm{O}_{3}\right)$ while others are increasing $\left(\mathrm{MnO}, \mathrm{K}_{2} \mathrm{O}\right)$. Many others are divergent, the highest concentration of some $\left(\mathrm{MnO}, \mathrm{TiO}_{2}, \mathrm{Al}_{2} \mathrm{O}_{3}\right)$ coinciding with the lowest of others $\left(\mathrm{K}_{2} \mathrm{O}, \mathrm{SiO}_{2}\right)$. However, there is no continuous evolution (increasing or decreasing), all the major oxide having a zigzag behaviour.

Table 2. Microanalysis (wt. \%) of kaolinite. Structural formula with 11 Oxygens

\begin{tabular}{|c|c|c|c|c|c|c|c|c|c|c|c|c|c|c|c|c|c|c|c|c|c|c|}
\hline Points & 1 & 2 & 3 & 4 & 5 & 6 & 7 & 8 & 9 & 10 & 11 & 12 & 13 & 14 & 15 & 16 & 17 & 18 & 19 & 20 & 21 & 22 \\
\hline \multicolumn{23}{|c|}{ Microanalysis } \\
\hline $\mathrm{F}$ & 0 & 0 & 0.147 & 0.221 & 0.441 & 0.221 & 2.959 & 0.147 & 0 & 0.148 & 0.734 & 0.148 & 0 & 0.658 & 0 & 0 & 0.295 & 0 & 0 & 0 & 0.149 & 2.529 \\
\hline $\mathrm{Na}_{2} \mathrm{O}$ & 0.011 & 0.011 & 0 & 0.03 & 0.028 & 0 & 0.062 & 0.044 & 0.074 & 0.013 & 0 & 0.034 & 0 & 0.008 & 0.067 & 0.1 & 0.027 & 0.101 & 0.094 & 0.027 & 0 & 0.049 \\
\hline \multirow[t]{2}{*}{$\mathrm{MgO}$} & 0.046 & 0 & 0.014 & 0.032 & 0.046 & 0.009 & 0.054 & 0.044 & 0.049 & 0.147 & 0.175 & 0.171 & 0.211 & 0.266 & 0.03 & 0.028 & 0.022 & 0.041 & 0.063 & 0.029 & 0.026 & 0.075 \\
\hline & & 33.64 & 36.24 & 35.64 & 35.71 & 34.65 & 34.40 & 34.07 & 34.22 & 37.19 & 35.76 & 36.62 & 36.38 & & 35.67 & 36.05 & 32.21 & 36.53 & 34.46 & 35.87 & 34.85 & 35.29 \\
\hline \multirow[t]{2}{*}{$\mathrm{Al}_{2} \mathrm{O}_{3}$} & 34.15 & 3 & 3 & 1 & 1 & 9 & 2 & 8 & 1 & 7 & 9 & 9 & 1 & 37.88 & 1 & 5 & 3 & 3 & 6 & 3 & 1 & 9 \\
\hline & 44.37 & 41.30 & 47.15 & 46.73 & 46.43 & 45.99 & & 45.93 & 46.28 & 47.25 & 46.65 & 48.10 & & 46.83 & 47.09 & 45.86 & 43.62 & & & 47.21 & 46.41 & 46.61 \\
\hline $\mathrm{SiO}_{2}$ & 4 & 9 & 8 & 1 & 8 & 3 & 45.78 & 7 & 5 & 3 & 6 & 5 & 48.78 & 4 & 8 & 2 & 8 & 48.01 & 46.33 & 7 & 8 & 1 \\
\hline $\mathrm{Cl}$ & 0.001 & 0.023 & 0.015 & 0.039 & 0.028 & 0.036 & 0.001 & 0.027 & 0.025 & 0.019 & 0.005 & 0.011 & 0.018 & 0.003 & 0.046 & 0.029 & 0.035 & 0.071 & 0.046 & 0.019 & 0.015 & 0.007 \\
\hline $\mathrm{K}_{2} \mathrm{O}$ & 0.015 & 0.052 & 0 & 0 & 0.012 & 0.039 & 0.056 & 0 & 0.06 & 0.014 & 0 & 0.021 & 0.008 & 0.015 & 0.017 & 0.041 & 0.075 & 0 & 0.06 & 0.019 & 0.033 & 0.035 \\
\hline $\mathrm{CaO}$ & 0.004 & 0.01 & 0.004 & 0.015 & 0.009 & 0.013 & 0 & 0.019 & 0.011 & 0.04 & 0.02 & 0.035 & 0.041 & 0.024 & 0.013 & 0.002 & 0.008 & 0.031 & 0.012 & 0.023 & 0.039 & 0.07 \\
\hline $\mathrm{TiO}_{2}$ & 0.043 & 0.08 & 0.027 & 0.021 & 0 & 0.021 & 0.094 & 0.033 & 0.071 & 0.001 & 0.001 & 0.029 & 0.036 & 0.014 & 0.261 & 0.014 & 0.002 & 0 & 0.038 & 0.147 & 0.603 & 0.296 \\
\hline $\mathrm{Cr}_{2} \mathrm{O}_{3}$ & 0.004 & 0.009 & 0.031 & 0.038 & 0.041 & 0.02 & 0.031 & 0.046 & 0.033 & 0.046 & 0 & 0.071 & 0.033 & 0.05 & 0.079 & 0.088 & 0.062 & 0.039 & 0.057 & 0.008 & 0.005 & 0.005 \\
\hline $\mathrm{MnO}$ & 0 & 0 & 0.002 & 0 & 0.048 & 0 & 0 & 0 & 0 & 0.038 & 0.053 & 0.022 & 0 & 0.01 & 0 & 0 & 0 & 0.005 & 0.053 & 0 & 0.046 & 0.037 \\
\hline $\mathrm{FeO}$ & 0.282 & 0.339 & 0.263 & 0.178 & 0.203 & 0.348 & 0.223 & 0.427 & 0.265 & 0.319 & 0.408 & 0.407 & 0.477 & 0.478 & 0.558 & 0.717 & 0.688 & 0.439 & 0.717 & 0.647 & 0.412 & 0.761 \\
\hline $\mathrm{NiO}$ & 0.01 & 0.051 & 0 & 0 & 0.019 & 0.044 & 0.019 & 0 & 0 & 0 & 0.012 & 0 & 0 & 0.041 & 0.039 & 0.002 & 0.012 & 0.041 & 0 & 0.049 & 0.019 & 0.066 \\
\hline $\mathrm{BaO}$ & 0.113 & 0.063 & 0.036 & 0.032 & 0 & 0 & 0.005 & 0.041 & 0 & 0 & 0 & 0.041 & 0.055 & 0 & 0 & 0.023 & 0 & 0 & 0 & 0 & 0.095 & 0 \\
\hline \multirow[t]{2}{*}{$\mathrm{SrO}$} & 0 & 0 & 0 & 0 & 0 & 0 & 0 & 0 & 0 & 0 & 0 & 0 & 0 & 0 & 0 & 0 & 0 & 0 & 0 & 0 & 0 & 0 \\
\hline & 79.05 & & & 82.97 & 83.02 & 81.40 & 83.68 & 80.84 & 81.09 & 85.23 & 83.83 & 85.72 & 86.03 & 86.28 & 83.87 & 82.96 & 77.06 & 85.31 & 81.93 & 84.05 & 82.71 & 85.83 \\
\hline Total & 3 & 75.59 & 83.94 & 9 & 6 & 2 & 6 & 2 & 4 & 5 & 3 & 5 & 9 & 2 & 9 & 2 & 7 & 3 & 7 & 8 & 2 & 9 \\
\hline \multicolumn{23}{|c|}{ Structural formula } \\
\hline $\mathrm{Na}$ & 0.002 & 0.002 & 0 & 0.004 & 0.004 & 0 & 0.009 & 0.006 & 0.01 & 0.002 & 0 & 0.004 & 0 & 0.001 & 0.009 & 0.014 & 0.004 & 0.013 & 0.013 & 0.004 & 0 & 0.013 \\
\hline
\end{tabular}




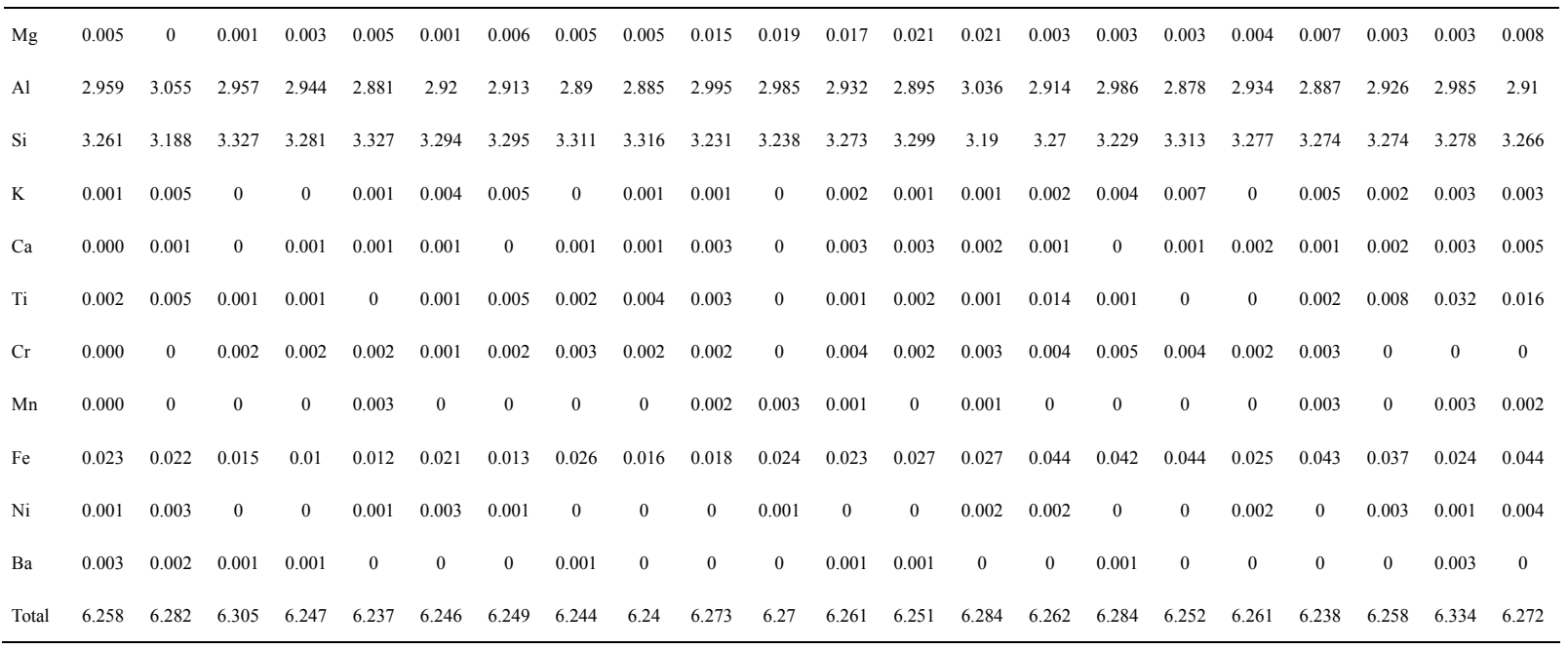

Table 3. Microanalysis (wt. \%) and structural formula of muscovite/illite (1-11) and anatase (A)

\begin{tabular}{|c|c|c|c|c|c|c|c|c|c|c|c|c|}
\hline Points & 1 & 2 & 3 & 4 & 5 & 6 & 7 & 8 & 9 & 10 & 11 & $\mathrm{~A}$ \\
\hline \multicolumn{13}{|c|}{ Microstructure } \\
\hline $\mathrm{F}$ & 0.146 & 0.144 & 0.22 & 0 & 0.223 & 0 & 0.3 & 0.15 & 0 & 0 & 0.223 & 0 \\
\hline $\mathrm{Na}_{2} \mathrm{O}$ & 0.116 & 0.136 & 0.155 & 0 & 0.058 & 0.234 & 0.396 & 0.293 & 0.257 & 0.17 & 0.079 & 0.021 \\
\hline $\mathrm{MgO}$ & 0.427 & 1.652 & 1.601 & 0.679 & 0.994 & 0.118 & 0.143 & 0.752 & 0.409 & 0.35 & 0.207 & 0.002 \\
\hline $\mathrm{Al}_{2} \mathrm{O}_{3}$ & 36.097 & 35.008 & 31.382 & 29.806 & 29.529 & 35.314 & 36.151 & 33.44 & 36.483 & 32.5 & 33.282 & 4.39 \\
\hline $\mathrm{SiO}_{2}$ & 48.361 & 46.315 & 50.674 & 45.256 & 51.166 & 49.982 & 49.928 & 48.429 & 48.337 & 49.109 & 48.12 & 0.419 \\
\hline $\mathrm{Cl}$ & 0.004 & 0 & 0.002 & 0.006 & 0 & 0.052 & 0.044 & 0 & 0.013 & 0.004 & 0.008 & 0 \\
\hline $\mathrm{K}_{2} \mathrm{O}$ & 9.693 & 10.198 & 9.933 & 9.436 & 10.086 & 9.208 & 8.743 & 9.308 & 8.824 & 9.148 & 6.179 & 0.076 \\
\hline $\mathrm{CaO}$ & 0.028 & 0.009 & 0.012 & 0.025 & 0.033 & 0.016 & 0 & 0 & 0 & 0 & 0 & 0.021 \\
\hline $\mathrm{TiO}_{2}$ & 0.113 & 0.179 & 0.078 & 0.202 & 0.202 & 0 & 0.004 & 0 & 0.016 & 0 & 0.023 & 85.198 \\
\hline $\mathrm{Cr}_{2} \mathrm{O}_{3}$ & 0 & 0 & 0 & 0.022 & 0 & 0.01 & 0.053 & 0 & 0 & 0 & 0.027 & 0.803 \\
\hline $\mathrm{MnO}$ & 0.033 & 0.074 & 0.008 & 0.065 & 0.051 & 0.024 & 0 & 0 & 0 & 0.081 & 0.051 & 0.054 \\
\hline $\mathrm{FeO}$ & 2.599 & 4.142 & 2.977 & 2.609 & 3.081 & 0.626 & 0.351 & 1.089 & 0.39 & 1.13 & 0.943 & 1.731 \\
\hline $\mathrm{NiO}$ & 0.036 & 0.091 & 0.005 & 0.007 & 0.046 & 0.029 & 0.012 & 0.046 & 0.019 & 0.019 & 0.034 & 0.018 \\
\hline $\mathrm{BaO}$ & 0.658 & 0.661 & 0.32 & 0.005 & 0 & 0.186 & 0 & 0.086 & 0.041 & 0.263 & 0.154 & 0 \\
\hline $\mathrm{SrO}$ & 0 & 0 & 0 & 0 & 0 & 0 & 0 & 0 & 0 & 0 & 0 & 0 \\
\hline Total & 98.311 & 98.609 & 97.368 & 88.117 & 95.467 & 95.799 & 96.126 & 93.592 & 94.789 & 92.775 & 89.33 & 92.733 \\
\hline \multicolumn{13}{|c|}{ Structural formula } \\
\hline $\mathrm{Na}$ & 0.015 & 0.017 & 0.02 & 0 & 0.007 & 0.029 & 0.049 & 0.038 & 0.033 & 0.022 & 0.01 & 0.001 \\
\hline $\mathrm{Mg}$ & 0.041 & 0.161 & 0.157 & 0.073 & 0.099 & 0.012 & 0.014 & 0.076 & 0.04 & 0.036 & 0.021 & 0 \\
\hline $\mathrm{Al}$ & 2.747 & 2.683 & 2.412 & 2.525 & 2.31 & 2.7 & 2.744 & 2.636 & 2.811 & 2.577 & 2.687 & 0.071 \\
\hline $\mathrm{Si}$ & 3.129 & 3.017 & 3.311 & 3.259 & 3.403 & 3.249 & 3.222 & 3.244 & 3.266 & 3.309 & 3.302 & 0.006 \\
\hline $\mathrm{K}$ & 0.8 & 0.848 & 0.828 & 0.867 & 0.856 & 0.764 & 0.72 & 0.796 & 0.738 & 0.787 & 0.541 & 0.001 \\
\hline $\mathrm{Ca}$ & 0.002 & 0.001 & 0.001 & 0.002 & 0.002 & 0.001 & 0 & 0 & 0 & 0 & 0 & 0 \\
\hline $\mathrm{Ti}$ & 0.005 & 0.009 & 0.004 & 0.011 & 0.01 & 0 & 0 & 0 & 0.001 & 0 & 0.001 & 0.92 \\
\hline $\mathrm{Cr}$ & 0 & 0 & 0 & 0.001 & 0 & 0.001 & 0 & 0 & 0 & 0 & 0.001 & 0.009 \\
\hline $\mathrm{Mn}$ & 0.002 & 0.004 & 0 & 0.004 & 0.003 & 0.001 & 0.004 & 0 & 0 & 0.005 & 0.003 & 0.001 \\
\hline $\mathrm{Fe}$ & 0.14 & 0.304 & 0.162 & 0.157 & 0.171 & 0.034 & 0.019 & 0.061 & 0.021 & 0.063 & 0.054 & 0.021 \\
\hline $\mathrm{Ni}$ & 0.002 & 0.005 & 0 & 0 & 0.002 & 0.002 & 0.018 & 0.002 & 0.001 & 0.001 & 0.002 & 0 \\
\hline $\mathrm{Ba}$ & 0.017 & 0.017 & 0.008 & 0 & 0 & 0.005 & 0 & 0.002 & 0.001 & 0.007 & 0.004 & 0 \\
\hline Total & 6.901 & 7.065 & 6.903 & 6.9 & 6.864 & 6.797 & 6.791 & 6.855 & 6.912 & 6.808 & 6.628 & 1.03 \\
\hline
\end{tabular}


Table 4. Major elements content (wt. \%) of Mayouom clays (1.d.: detection limit)

\begin{tabular}{|c|c|c|c|c|c|c|c|c|c|}
\hline & \multirow{2}{*}{$\frac{\text { Sandy clays }}{\text { MY22S }}$} & \multicolumn{6}{|c|}{ Sand-poor clays } & \multirow{2}{*}{$\begin{array}{c}\text { Theorical pure } \\
\text { Kaolinite }\end{array}$} & \multirow{2}{*}{$\begin{array}{l}\text { Mylonitic host } \\
\text { rock }\end{array}$} \\
\hline & & MY22G & MY24 & MY25 & MY26 & MY27 & MY28 & & \\
\hline $\mathrm{SiO}_{2}$ & 65.86 & 43.23 & 43.3 & 45.73 & 45.89 & 43.41 & 44.22 & 46.55 & 63.3 \\
\hline $\mathrm{Al}_{2} \mathrm{O}_{3}$ & 22.60 & 35.19 & 34.68 & 34.14 & 34.73 & 35.47 & 34.4 & 39.49 & 15.2 \\
\hline $\mathrm{Fe}_{2} \mathrm{O}_{3}$ & 0.82 & 1.18 & 1 & 0.92 & 0.89 & 1.04 & 1.06 & 0 & 8.11 \\
\hline $\mathrm{MnO}$ & 0.002 & 0.009 & 0.013 & 0.009 & 0.008 & 0.019 & 0.027 & 0 & 0.11 \\
\hline $\mathrm{MgO}$ & 0.04 & 0.12 & 0.15 & 0.13 & 0.13 & 0.17 & 0.18 & 0 & 1.65 \\
\hline $\mathrm{CaO}$ & $<$ l.d. & $<$ l.d. & 0.02 & $<$ l.d. & $<$ l.d. & $<$ l.d. & $<$ l.d. & 0 & 6.1 \\
\hline $\mathrm{Na}_{2} \mathrm{O}$ & $<$ l.d. & $<$ l.d. & $<$ l.d. & $<$ l.d. & $<$ l.d. & $<$ l.d. & $<$ l.d. & 0 & 3.86 \\
\hline $\mathrm{K}_{2} \mathrm{O}$ & 0.40 & 0.57 & 0.5 & 0.78 & 0.76 & 0.93 & 0.67 & 0 & 0.64 \\
\hline $\mathrm{TiO}_{2}$ & 0.90 & 3.73 & 3.74 & 3.43 & 3 & 4.08 & 4.05 & 0 & 0.64 \\
\hline $\mathrm{P}_{2} \mathrm{O}_{5}$ & 0.11 & 0.21 & 0.29 & 0.21 & 0.16 & 0.22 & 0.26 & 0 & 0.28 \\
\hline LOI & 9.43 & 15.75 & 16.45 & 14.68 & 14.45 & 14.84 & 15.43 & 13.96 & 0.28 \\
\hline Total & 100.16 & 99.99 & 100.15 & 100.02 & 100 & 100.17 & 100.31 & 100 & 100.2 \\
\hline $\mathrm{SiO}_{2} / \mathrm{Al}_{2} \mathrm{O}_{3}$ & 2.91 & 1.23 & 1.25 & 1.34 & 1.32 & 1.22 & 1.29 & 1.18 & 4.16 \\
\hline
\end{tabular}

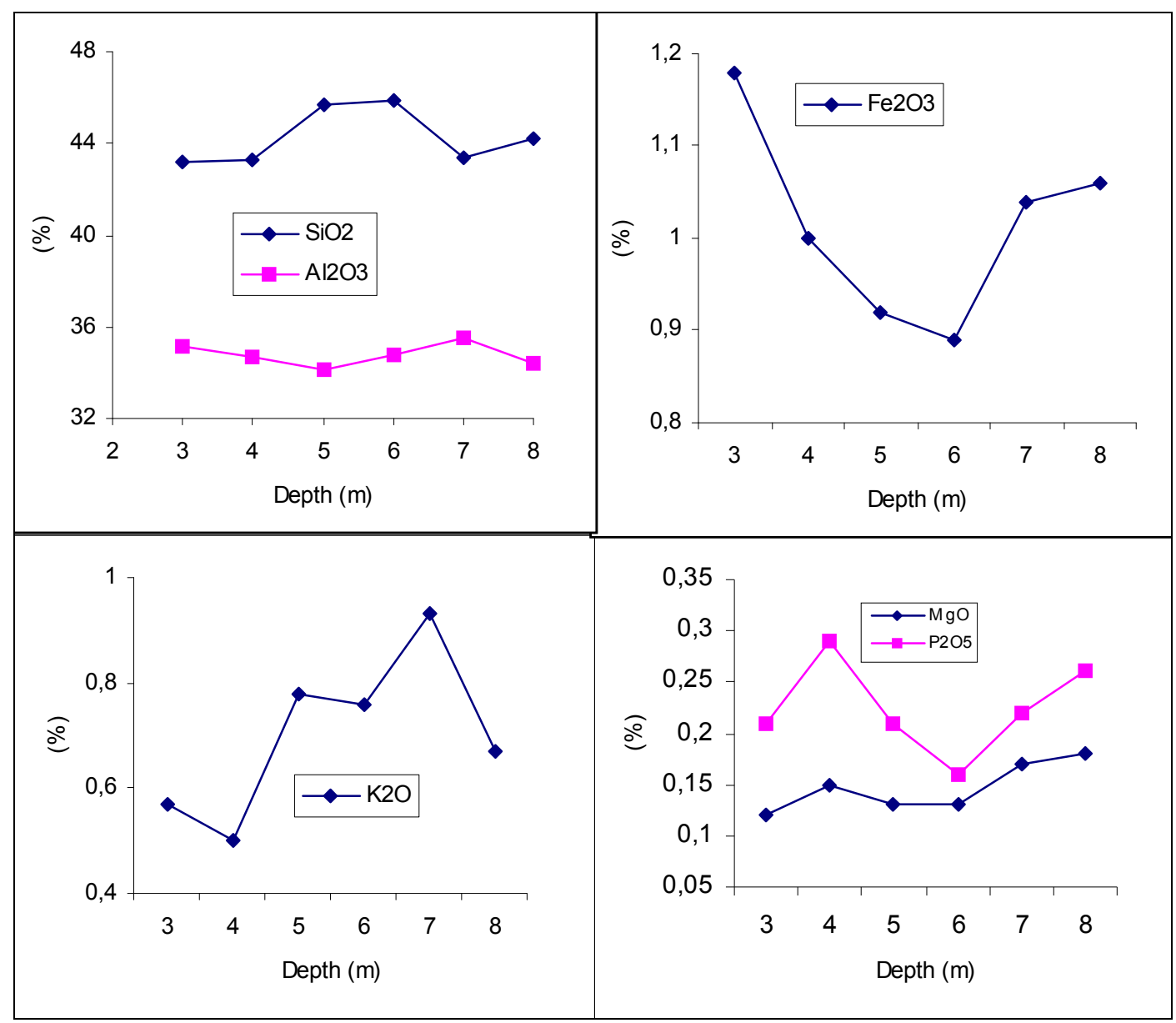

Figure 4. Example of low downwards variation of major elements content 
For trace element compositions of studied materials (Table 5), some trends are present. All the samples have medium content of $\mathrm{Ba}(113-259 \mathrm{ppm}), \mathrm{Sr}(56.1-253 \mathrm{ppm}), \mathrm{Zr}$ (125-754 ppm) and La (31.2-106 ppm) display wider interval of variation. These elements are associated to accessory minerals such as Ba, Sr-hydroxylapatite. Ce content is medium to high (65.2-204 ppm). Transition elements have low concentrations, except Cr (153-560 $\mathrm{ppm}$ ) and V (83.7-293 ppm). However, sand-poor kaolin samples in the other hand display higher content of $\mathrm{Cr}$ $(278-560 \mathrm{ppm})$ and V (207-293 ppm) than sandy kaolins (153 and 83.7 respectively) and no associated mineral has been identified. Toxic elements are absent $(\mathrm{As}, \mathrm{Cd}, \mathrm{Sb})$ or present with low content $(\mathrm{Pb}=5.98-25.1 \mathrm{ppm})$. When sand poor kaolins are compared as function of depth, some grouping can be done:

- Transition elements content (Ni, Co, V) show a general continuous increasing (Figure 5);

- $\mathrm{U}$ and $\mathrm{Pb}$ decrease from four metres downwards;

- Th and Cr have opposite behaviour: Th increases when $\mathrm{Cr}$ is decreasing, and at $6 \mathrm{~m}$, there is reversion.

- All other element display zigzag behaviour.

All these variations are displayed in relatively narrow intervals (1.38-4.30 for Co; $0.89-3.13$ for U; 278-560 for $\mathrm{Cr}$...), indicating that the material is relatively homogenous in all its thickness.

Table 6 presents the Rare Earth Elements (REE) content and their pattern, normalised to chondrite CI values of Mc Donough and Sun (1995) are shown in Figure 6. All the kaolin samples show similar REE patterns, parallel to each other; being more enriched in Light Rare Earth Element (LREE) compared to Heavy Rare Earth Element (HREE). Sandy kaolin (MY22S) displays the highest $\mathrm{LaN} / \mathrm{YbN}$ ratio. A distinct negative Eu anomaly $\left(\mathrm{Eu} / \mathrm{Eu}^{*}=\mathrm{EuN} /(\mathrm{SmN} \times \mathrm{GdN})^{1 / 2}=0.595\right)$ is observed in sandy clays. The behaviour of that element is related to that of $\mathrm{Ca}$ and $\mathrm{Na}$ during alteration of feldspars. None sample shows $\mathrm{Ce}$ anomaly.

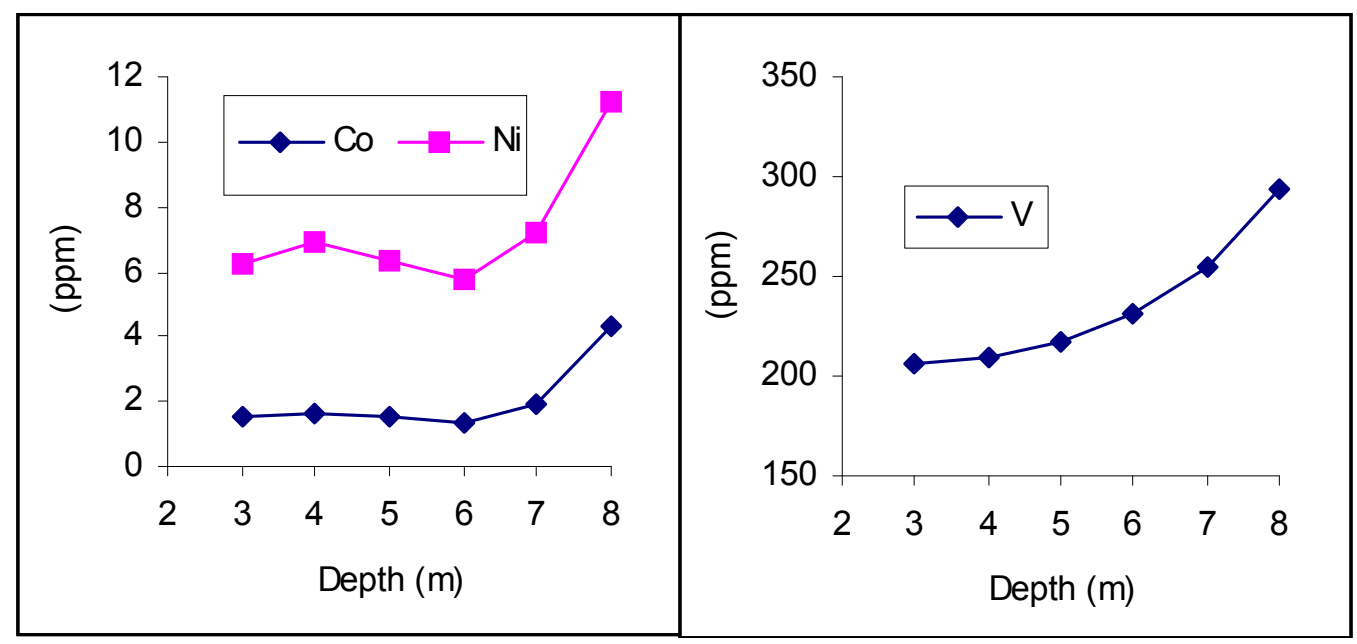

Figure 5. Continuous downward increasing of some trace elements 
Table 5. Trace elements content (wt. ppm); * is the mylonitic mother rock assigned to sandy clays (1.d. : detection limit; nd: not determined)

\begin{tabular}{|c|c|c|c|c|c|c|c|c|}
\hline & MY22S & MY22G & MY24 & MY25 & MY26 & MY27 & MY28 & Parent rock* \\
\hline As & $<$ l.d. & < l.d. & < l.d. & < l.d. & < l.d. & 1.49 & 1.09 & nd \\
\hline $\mathrm{Ba}$ & 156 & 113 & 219 & 258 & 156 & 189 & 259 & 161 \\
\hline $\mathrm{Be}$ & 0.69 & 0.74 & 0.89 & 0.92 & 0.79 & 0.69 & 0.99 & nd \\
\hline $\mathrm{Bi}$ & $<$ l.d. & $<$ l.d. & $<$ l.d. & $<$ l.d. & $<$ l.d. & $<$ l.d. & $<$ l.d. & nd \\
\hline $\mathrm{Cd}$ & $<$ l.d. & $<$ l.d. & $<$ l.d. & $<$ l.d. & $<$ l.d. & $<$ l.d. & $<$ l.d. & nd \\
\hline $\mathrm{Ce}$ & 165 & 68.8 & 204 & 136 & 67.1 & 65.2 & 149 & 39.3 \\
\hline Co & 0.6 & 1.54 & 1.62 & 1.5 & 1.38 & 1.96 & 4.3 & 44.6 \\
\hline $\mathrm{Cr}$ & 153 & 560 & 482 & 361 & 278 & 500 & 452 & 19 \\
\hline Cs & 0.5 & 0.82 & 73 & 0.89 & 0.81 & 0.79 & 0.82 & nd \\
\hline $\mathrm{Cu}$ & 16.4 & 21.9 & 38.6 & 19.1 & 22 & 19.9 & 72.2 & 19.7 \\
\hline Dy & 3.94 & 2.27 & 5.91 & 4.48 & 2.33 & 2.31 & 4.26 & 2.8 \\
\hline $\mathrm{Er}$ & 1.25 & 0.946 & 1.94 & 1.57 & 0.97 & 1.09 & 1.48 & 1.55 \\
\hline $\mathrm{Eu}$ & 1.75 & 1.26 & 3.59 & 2.76 & 1.45 & 1.42 & 2.92 & 1.78 \\
\hline $\mathrm{Ga}$ & 27.2 & 40.4 & 39.5 & 43.7 & 41.4 & 44 & 43 & nd \\
\hline Gd & 7.25 & 3.29 & 9.23 & 6.98 & 3.39 & 3.18 & 6.98 & 3.48 \\
\hline $\mathrm{Ge}$ & 1.38 & 1.89 & 2.32 & 1.93 & 2.01 & 2.09 & 2.26 & nd \\
\hline $\mathrm{Hf}$ & 8.25 & 5.59 & 5.6 & 8.04 & 7.16 & 6.78 & 7.24 & nd \\
\hline Но & 0.56 & 0.359 & 0.869 & 0.669 & 0.375 & 0.383 & 0.619 & nd \\
\hline In & 0.08 & 0.17 & 0.16 & 0.17 & 0.16 & 0.17 & 0.18 & nd \\
\hline $\mathrm{La}$ & 81.8 & 34.4 & 106 & 69.2 & 33.4 & 31.2 & 72.5 & 18.3 \\
\hline $\mathrm{Lu}$ & 0.106 & 0.145 & 0.195 & 0.182 & 0.153 & 0.201 & 0.212 & 0.26 \\
\hline Mo & 2.8 & 2.8 & 2.92 & 2.4 & 2.17 & 3.11 & 3.1 & $\mathrm{nd}$ \\
\hline $\mathrm{Nb}$ & 11.4 & 34.5 & 34.4 & 45.6 & 35.7 & 44.3 & 47.7 & nd \\
\hline $\mathrm{Nd}$ & 66 & 26.2 & 77.9 & 56.6 & 27.8 & 27.7 & 65.2 & 19.3 \\
\hline $\mathrm{Ni}$ & 4.5 & 6.2 & 6.9 & 6.3 & 5.8 & 7.2 & 11.2 & 18.6 \\
\hline $\mathrm{Pb}$ & 21.7 & 11.1 & 25.1 & 11.1 & 7.88 & 5.98 & 6.97 & nd \\
\hline $\operatorname{Pr}$ & 18.4 & 7.48 & 22.1 & 15.6 & 7.52 & 7.5 & 17.8 & nd \\
\hline $\mathrm{Rb}$ & 12.6 & 36 & 36.9 & 40.6 & 40.6 & 50.1 & 34.8 & nd \\
\hline Sb & < l.d. & < l.d. & $<$ l.d. & < l.d. & $<$ l.d. & $<$ l.d. & $<$ l.d. & nd \\
\hline $\mathrm{Sm}$ & 11.1 & 4.68 & 12.9 & 9.8 & 4.86 & 4.96 & 10.9 & 3.73 \\
\hline Sn & 1.75 & 2.17 & 3.56 & 2.62 & 2.1 & 2.54 & 4.66 & $\mathrm{nd}$ \\
\hline $\mathrm{Sr}$ & 96.2 & 64.4 & 253 & 155 & 61.1 & 56.1 & 184 & 434 \\
\hline $\mathrm{Ta}$ & 0.48 & 2.34 & 2.32 & 2.8 & 2.21 & 3.03 & 3.19 & nd \\
\hline $\mathrm{Tb}$ & 0.884 & 0.454 & 1.23 & 0.928 & 0.457 & 0.455 & 0.935 & nd \\
\hline Th & 27.8 & 2.39 & 2.78 & 4.65 & 5.95 & 3.25 & 3.41 & nd \\
\hline $\mathrm{Tm}$ & 0.135 & 0.13 & 0.217 & 0.192 & 0.136 & 0.167 & 0.195 & nd \\
\hline $\mathrm{U}$ & 1.9 & 2.32 & 3.13 & 2.38 & 2.4 & 1.14 & 0.89 & nd \\
\hline V & 83.7 & 207 & 210 & 217 & 231 & 254 & 293 & 95.8 \\
\hline W & < l.d. & 0.35 & 0.48 & 0.35 & 0.36 & 0.44 & 0.46 & nd \\
\hline $\mathrm{Y}$ & 12.6 & 7.71 & 17.3 & 13.9 & 8.79 & 8.84 & 12.9 & 16.7 \\
\hline $\mathrm{Yb}$ & 0.786 & 0.896 & 1.32 & 1.21 & 0.964 & 1.24 & 1.37 & 1.52 \\
\hline $\mathrm{Zn}$ & 16.1 & 46.9 & 56.3 & 43.4 & 35.4 & 46.3 & 55.9 & 94.6 \\
\hline $\mathrm{Zr}$ & 325 & 232 & 239 & 353 & 318 & 304 & 319 & 25.7 \\
\hline
\end{tabular}




\subsection{Quantitative Mineralogy}

The modal compositions obtain from calculations are presented in Table 7. The sum of reconstituted minerals percentage is equal to $100 \%$. These results show that our clays are essentially composed of Kaolinite (54-84\%) associated to quartz, muscovite, anatase, hydroxylapatite and hematite. The sandy sample MY22S is similar to other sandy clays of the deposit (Njoya, 2004). This sample is totally different from sand-poor clays with it kaolinite and quartz percentage.

Table 6. Rare Earth Element contents (wt. ppm) of the studied sample

\begin{tabular}{|c|c|c|c|c|c|c|c|c|c|}
\hline & MY22S & MY22G & MY24 & MY25 & MY26 & MY27 & MY28 & CI (M\&S) & Mantle (M\&S) \\
\hline La & 81.8 & 34.4 & 106 & 69.2 & 33.4 & 31.2 & 72.5 & 0.237 & 0.648 \\
\hline $\mathrm{Ce}$ & 163 & 68.8 & 204 & 136 & 33.4 & 63.2 & 149 & 0.613 & 1.675 \\
\hline Pr & 18.4 & 7.48 & 22.1 & 15.6 & 67.1 & 7.5 & 17.8 & 0.0928 & 0.254 \\
\hline Nd & 66 & 262 & 77.9 & 56.6 & 7.52 & 27.7 & 65.2 & 0.457 & 1.25 \\
\hline Sm & 11.1 & 4.68 & 12.9 & 9.8 & 27.8 & 4.96 & 10.9 & 0.148 & 0.406 \\
\hline Eu & 1.75 & 1.26 & 3.59 & 2.76 & 4.86 & 1.42 & 2.92 & 0.0563 & 0.154 \\
\hline Gd & 7.25 & 3.29 & 9.23 & 6.98 & 1.45 & 3.18 & 6.98 & 0.199 & 0.544 \\
\hline $\mathbf{T b}$ & 0.884 & 0.545 & 1.23 & 0.928 & 3.39 & 0.455 & 0.935 & 0.0361 & 0.099 \\
\hline Dy & 3.94 & 2.27 & 5.91 & 4.48 & 0.457 & 2.31 & 4.26 & 0.246 & 0.674 \\
\hline Ho & 0.56 & 0.359 & 0.869 & 0.669 & 2.33 & 0.383 & 0.619 & 0.0546 & 0.149 \\
\hline $\mathbf{E r}$ & 1.25 & 0.946 & 1.94 & 1.57 & 0.375 & 1.09 & 1.48 & 0.16 & 0.438 \\
\hline Tm & 0.135 & 0.13 & 0.217 & 0.192 & 0.97 & 0.167 & 0.195 & 0.0247 & 0.068 \\
\hline $\mathbf{Y b}$ & 0.786 & 0.896 & 1.32 & 1.21 & 0.136 & 1.24 & 1.37 & 0.161 & 0.441 \\
\hline Lu & 0.106 & 0.145 & 0.195 & 0.182 & 0.961 & 0.201 & 0.212 & 0.0246 & 0.0675 \\
\hline $\mathrm{CeN} / \mathrm{YbN}$ & 70.698 & 26.081 & 54.552 & 38.851 & 166.834 & 17.093 & 35.95 & & \\
\hline $\mathbf{L a N} / \mathbf{Y b N}$ & 54.467 & 20.167 & 40.59 & 29.52 & 64.502 & 13.386 & 28.565 & & \\
\hline $\mathrm{Ce} / \mathrm{Ce}^{*}$ & 1.029 & 1.038 & 1.02 & 1.001 & 1.024 & 1.031 & 1.003 & & \\
\hline $\mathbf{E u} / \mathbf{E u}$ * & 0.595 & 0.979 & 1.003 & 1.017 & 1.089 & 1.09 & 1.02 & & \\
\hline
\end{tabular}

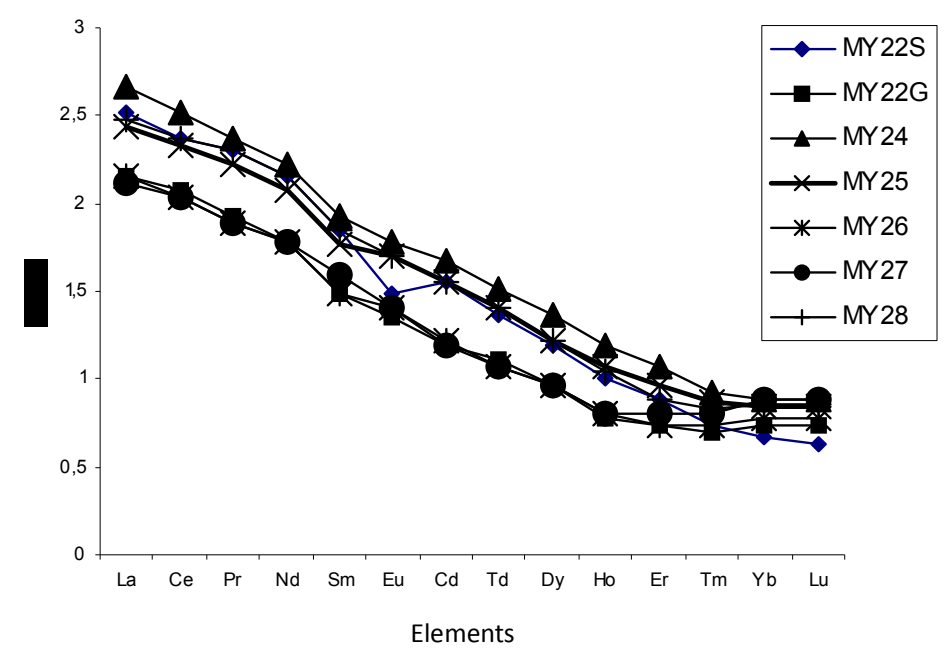

Figure 6. Rare Earth Element patterns. Observe the Eu negative anomaly in MY22S 
Table 7. Quantitative mineralogical composition (wt. \%) of the studied materials, the average and "écart-type" of sand poor samples

\begin{tabular}{lccccccccc}
\hline & MY22S & MY22G & MY24 & MY25 & MY26 & MY27 & MY28 & $\begin{array}{c}\text { average } \\
\text { sand-poor }\end{array}$ & $\begin{array}{c}\text { Standard } \\
\text { deviation }\end{array}$ \\
\hline Muscovite & 3.5 & 4.82 & 4.24 & 6.6 & 6.44 & 7.88 & 5.68 & 5.94 & 1.32 \\
Kaolinite & $\mathbf{5 3 . 7 6}$ & 84.32 & 83.6 & 79.93 & 81.58 & 82.04 & 81.49 & 82.16 & 1.58 \\
Hydroxylapatite & 0.76 & 1.46 & 2.01 & 1.46 & 1.12 & 1.53 & 1.81 & 1.57 & 0.31 \\
Quartz & $\mathbf{3 9 . 2 7}$ & 1.83 & 2.5 & 5.57 & 5.03 & 1.68 & 3.75 & 3.39 & 1.66 \\
Hematite & 0.82 & 1.18 & 1 & 0.92 & 0.89 & 1.04 & 1.06 & 1.02 & 0.1 \\
Anatase & 0.9 & 3.73 & 3.74 & 3.43 & 3 & 4.08 & 4.05 & 3.72 & 0.44 \\
Indeterminate & 0.99 & 2.66 & 2.91 & 2.09 & 1.95 & 1.73 & 2.16 & 2.25 & 0.45 \\
Total & 100.00 & 100.00 & 100.00 & 100.00 & 100.00 & 100.00 & 100.00 & & \\
\hline
\end{tabular}

\section{Discussion}

On the field, sandy clays present marks of tectonics deformation of mylonites. This implies that the structure of the basement rock plays an important role during kaolinization, notably in the circulation of the magmatic fluids (Njoya, 2006). The sandy sample is the transformation product of the mylonites. In sand-poor clays, relic of muscovite and large crystals of kaolinite formed from the pseudomorphose of feldspars imply that their parent rock could be either granular or porphyritic microlitic rock. The basalts around the deposit are porphyritic microlitic in texture. The conservation of the structure of the basement rock and the shape of quartz crystals can be interpreted as prove of in situ alteration. The presence of such quartz particles is helpful in abrasive usage of clays (Pruett \& Murray, 1991).

Chemically, the clays are $\mathrm{SiO}_{2}, \mathrm{Al}_{2} \mathrm{O}_{3}$ and LOI rich, while $\mathrm{MnO}, \mathrm{CaO}$ and $\mathrm{Na}_{2} \mathrm{O}$ are completely leached. This leaching corresponds to the higher mobility of those oxides during kaolinization process and it is compatible with an advance argillic alteration system close to hydrothermal kaolin deposits (Meyer \& Hemley, 1967; Meunier et al., 1983; Inoue, 1995; Dill et al., 1995, 1997, 2000). The higher concentration of Cr (278-560 ppm) and V (207-293 ppm) in sand-poor kaolin samples without any associated mineral may be related to their low mobility during kaolinization process, and to enrichment of these elements in parent intrusive veins (Bariand et al., 1985; Vidal, 1998).

Mineralogical analyses reveal the remarkable predominance of Kaolinite, associated to muscovite, quartz, anatase, Hydroxylapatite and hematite. Such mineral assemblage is common in both meteoric and hydrothermal clay deposits. However in humid tropical climate, meteoric weathering generally leads to formation of kaolinite with poor crystallinity due to numerous impurities as $\mathrm{Fe}, \mathrm{Ti}, \mathrm{Cr}$ and $\mathrm{Ni}$ in kaolinite structure (Braun et al., 1990, 1998; Petit, 1994). The Kaolinite from Mayouom deposit shows good crystallinity, excluding a meteoric process of weathering. The higher Kaolinite content of these clay materials (except MY22S that will need some elimination of quartz), make them good clays for paints and ceramics (Njoya, 2004). This kaolinite content is linked to the high feldspars content of the mother rock. Anatase and hematite can be considered as precipitation of oxides during alteration of ferrotitanium minerals. Considering the sand-poor samples from 3 to $8 \mathrm{~m}$ depth with their "ecart-type" (from 0.10 for hematite to 1.58 for Kaolinite), the deposit can be considered as vertically homogeneous in term of quantitative and qualitative mineralogical composition.

Most of the trace elements contents are low. Comparison of MY22S with Mayouom mylonites (from Njongfang, 1998) reveals the impoverishment in $\mathrm{Eu}, \mathrm{Er}, \mathrm{Yb}, \mathrm{Lu}, \mathrm{Ni}, \mathrm{Co}, \mathrm{Cu}, \mathrm{Y}, \mathrm{Zn}$ and $\mathrm{Sr}$ during alteration and kaolinization process. Sr is totally absent while $\mathrm{Na}$ and $\mathrm{Ca}$ are fewly represented. In fact, during alteration process, cations of smaller radius (such as $\mathrm{Ca}, \mathrm{Na}, \mathrm{Sr}$ ) are easily leached out (Nyakairu et al., 2001; Nesbitt et al., 1980; Wronkiewicz \& Condie, 1987), this can be explain by the higher alterability of plagioclases in the mother rock, and also the advanced level of alteration in the deposit (Nesbitt et al., 1980; Wronkiewicz \& Condie, 1987).

The REE patterns are parallel, suggesting that all the clays were formed through the same process. The absence of $\mathrm{Ce}$ anomaly $\left(0.9<\mathrm{Ce} / \mathrm{Ce}^{*}<1.1\right)$ in all samples confirm that the kaolinization has taken place in a hydrothermal milieu. In such milieu, the absence of $\mathrm{Ce}$ anomaly implies the ultimate cooling stage characterised by the abundance of phyllitous mineral (Kaolinite, muscovite). 
The Eu negative anomaly $\left(\mathrm{Eu} / \mathrm{Eu}^{*}=0.6\right)$ in MY22S shows that the behaviour of that element may be related to that of $\mathrm{Ca}$ and $\mathrm{Na}$ (reduction from $\mathrm{Eu}^{3+}$ to $\mathrm{Eu}^{2+}$ ) during alteration process of feldspars in acid rocks. The mineral assemblage is that of veins, telescopes, small metallic deposits and precious stones, usually associated with granite, granodiorite shear zone and some volcanic areas (Barnes, 1967; Dill et al., 1995; Corbett, 2002). This can explain the high content of $\mathrm{Ba}, \mathrm{Sr}, \mathrm{V}$, and $\mathrm{Zr}$, element of high commercial value, in Mayouom clay.

The low vertical variation of major and trace elements, and that of qualitative and quantitative mineralogical composition is helpful during industrial exploitation for, in this deposit, the quality of clays is not influenced by the depth and therefore, the same treatment conditions can be used for all the material without any need of change due to variation of quality.

Comparison of these clays with those from other African countries such as Calabar in Nigeria (Njopwouo et al., 1998), Buwambo and Migrade in Uganda (Nyakairu et al., 2001) and worldwide (Cravero et al., 2010; Njoya, 2006, 2007; Nyakairu et al., 2001), Mayouom clays present suitable characteristics for industrial exploitation. In the ternary diagram of Fiori et al. (1989) in Türkmenoglu and Yavuz-Isik (2008), the clay materials from Mayouom correspond to suitable clays for white colored stoneware tiles except the sandy sample (MY22S) that occupy a position close to the composition favourable for making clinker (Türkmenoglu \& Yavuz-Isik, 2008). Also, the price of kaolin products is determined by its grade (Gougazeh \& Buhl, 2010). The Mayouom kaolin has low-screen residue value and more than $88 \%$ of particles are less than $63 \mu \mathrm{m}$ (Njoya, 2007). This makes Mayouom kaolin good materials for sanitary and pottery industries. However, the estimation of approximate total weight of the deposit needs a motorised drilling in order to know the real thickness from point to point.

\section{Conclusions}

The main outcomes of this paper are:

(1) Clays are $\mathrm{SiO}_{2}, \mathrm{Al}_{2} \mathrm{O}_{3}$ and $\mathrm{LOI}$ rich, with low CIA $\mathrm{Si}_{2} / \mathrm{Al}_{2} \mathrm{O}_{3}$, indicative of advanced alteration level;

(2) The predominant clay mineral is Kaolinite (up to $84 \%$ ), associated to quartz + illite/muscovite + anatase \pm hematite $\pm \mathrm{Ba}$, Sr-hydroxyapatite;

(3) In comparison to industrial clays from other African countries and worldwide, Mayouom clays present suitable characteristics for industrial exploitation: the sand-poor clays are very good materials for white colored stoneware tiles and other fine ceramics, paper coating and paint, while the sandy clays are appropriate for making clinker. Consequently, the studied clays are raw materials that can be highly recommended to clay consumer industries. Moreover, the advanced argillic alteration mineral assemblage can be considered as a rough indicator for exploration of related ores in neighbouring areas and along the northern Foumban shear zone.

\section{Acknowledgement}

All the analyses in this work were performed in CRPG (Nancy, France) through the financial support program "Coopération pour la Recherche Universitaire et Scientifique (CORUS 02412 129/24)". The authors are grateful to all the anonymous reviewers, for constructive comments on the manuscript. Especial thank goes to Dr A. S. L. Wouatong for his assistance.

\section{References}

Bariand, P., Cesbron, F., \& Geffroy, J. (1985). Les minéraux: leurs gisements, leurs associations (pp. 490). France: BRGM.

Barnes, H. L. (Ed.). (1967). Geochemistry of hydrothermal ore deposits.

Bloodworth, A. J., Highley, D. E., \& Mitchell, C. J. (1993). Industrial Minerals Laboratory Manual: Kaolin.

Braun, J. J., Pagel, M., Muller, J. P., Bilong, P., Michard, A., \& Guillet, B. (1990). Cerium anomalies in lateritic $\begin{array}{llll}\text { profiles. } \quad \text { Geochimica } & \text { 78ta, }\end{array}$ http://dx.doi.org/10.1016/0016-7037(90)90373-S

Braun, J. J., Viers, J., Dupré, B., Polve, M., Ndam, J., \& Muller, J. P. (1998). Solid/liquid REE fractionation in the lateritic system of Goyoum, East Cameroon: the implication for the present dynamics of the soil covers of the humid tropical regions. Geochimica et Cosmochimica Acta, 62(2), 273-299. http://dx.doi.org/10.1016/S0016-7037(97)00344-X

Corbett, G. (2002). Epithermal gold for explorationists. Abstract for AIG Journal, Paper, 1.

Cravero, F., Domínguez, E., \& Iglesias, C. (2001). Genesis and applications of the Cerro Rubio kaolin deposit, $\begin{array}{lllll}\text { Patagonia (Argentina). Applied clay 187-172. } & \text { 18(3), }\end{array}$ http://dx.doi.org/10.1016/S0169-1317(00)00021-1 
Cravero, F., Marfil, S. A., \& Maiza, P. J. (2010). Statistical analysis of geochemical data: a tool for discriminating between kaolin deposits of hypogene and supergene origin, Patagonia, Argentina. Clay Minerals, 45, 183-196. http://dx.doi.org/10.1180/claymin.2010.045.2.183

Dill, H. G., Bosse, H. R., Henning, K. H., Fricke, A., \& Ahrendt, H. (1997). Mineralogical and chemical variations in hypogene and supergene kaolin deposits in a mobile fold belt the Central Andes of northwestern Peru. Mineralium Deposita, 32(2), 149-163. http://dx.doi.org/10.1007/s001260050081

Dill, H. G., Bosse, H. R., \& Kassbohm, J. (2000). Mineralogical and chemical studies of volcanic-related argillaceous industrial minerals of the Central American Cordillera (Western El Salvador). Economic Geology, 95(3), 517-538.

Dill, H. G., Fricke, A., Henning, K. H., \& Theune, C. H. (1995). Aluminium phosphate mineralization from the hypogene La Vangardia kaolin deposit (Chile). Clay Minerals, 30, 249-256. http://dx.doi.org/10.1180/claymin.1995.030.3.08

Fiori, C., Fabbri, B., Donati, G., \& Venturi, I. (1989). Mineralogical composition of the clay bodies used in the Italian tile industry. Applied clay science, 4(5), 461-473. http://dx.doi.org/10.1016/0169-1317(89)90023-9

Gougazeh, M., \& Buhl, J. C. (2010). Geochemical and mineralogical characterization of the Jabal Al-Harad kaolin deposit, southern Jordan, for its possible utilization. Clay Minerals, 45(3), 301-314. http://dx.doi.org/10.1180/claymin.2010.045.3.301

Inoue, A. (1995). Formation of clay minerals in hydrothermal environment. In B. Velde (Ed.), Origin and Mineralogy of Clays: Clays and the Environment (pp. 268-329). Berlin: Springer. http://dx.doi.org/10.1007/978-3-662-12648-6_7

Martin, F. (2005). Le talc fait la résistance. J. CNRS, 181, 11.

McDonough, W. F., \& Sun, S. S. (1995). The composition of the Earth. Chemical geology, 120(3), 223-253. http://dx.doi.org/10.1016/0009-2541(94)00140-4

Merabet, D., \& Belkacemi, H. (2003, October). Caractérisation minéralogique et chimique du kaolin de Tamazert (Algérie). In Annales de Chimie Science des Matériaux (Vol. 28, No. 5, pp. 61-83). No longer published by Elsevier. http://dx.doi.org/10.1016/S0151-9107(03)00107-7

Meunier, A., Velde, B., Dudoignon, P., \& Beaufort, D. (1983). Identification of weathering and hydrothermal alteration in acidic rocks: petrography and mineralogy of clay minerals. Sci. Géol., Mém, 72, 93-99.

Meyer, C., \& Hemley, J. J. (1967). Wall rock alteration. In H. L. Barnes (Ed.), Geochemistry of Hydrothermal Ore Deposits (pp. 167-235).

Mitchell, C. J. (1994). Laboratory evaluation of kaolin: a case study from Zambia. In M. K. G. Whateley, \& P. K. Harvey (Eds.), Mineral Resource Evaluation II: Methods and Case HistoriesGeol Soci. Special Publ., 79, 241-247. http://dx.doi.org/10.1144/GSL.SP.1994.079.01.21

Murray, H. H., \& Keller, W. D. (1993). Kaolin, kaolin and kaolin. In H. H. Murray, W. Bondy, \& C. Harvey (Eds.), Kaolin Genesis and utilization. Special Publ., vol. 1.

Nesbitt, H. W., \& Markovics, G. (1980). Chemical processes affecting alkalis and alkaline earths during continental weathering. Geochimica et Cosmochimica Acta, 44(11), 1659-1666. http://dx.doi.org/10.1016/0016-7037(80)90218-5

Ngon, G. F., Etame, J., Ntamak-Nida, M. J., Mbog, M. B., Mpondo, A. M. M., Gérard, M., ... Bilong, P. (2012). Geological study of sedimentary clayey materials of the Bomkoul area in the Douala region (Douala sub-basin, Cameroon) for the ceramic industry. Comptes Rendus Geoscience. http://dx.doi.org/10.1016/j.crte.2012.05.004

Njonfang, E. (1998). Contribution à l'étude de la relation entre la Ligne du Cameroun et la Direction de l'Adamaoua. 1. Pétrologie, géochimie et structure des granitoïdes panafricains de la zone de cisaillement de Foumban-Bankim (Ouest-Cameroun et Adamaoua) 2. Pétrologie et géochimie des formations magmatiques tertiaires associées (Doctoral dissertation, Thèse Doct. Etat, Univ. Yaoundé I, Cameroun).

Njonfang, E., Moreau, C., \& Tchoua, M. F. (1998). La bande mylonitique Foumban-Bankim, Ouest-Cam. Une zone de cisaillement de Haute Température. C.R. Acad. Sci. Paris, 327, 735-741.

Njopwouo, D. (1984). Minéralogie et physico-chimie des argiles de Bamkoul et de Balengou (Cameroun). Utilisation dans la polymérisation du styrène et dans le renforcement du caoutchouc naturel (Doctor 
theory). University of Yaoundé, Cameroun.

Njopwouo, D., Tejiogap, E., Sondag, F., Volkoff, B., \& Wandji, R. (1998). Caractérisations minéralogiques et chimiques des argiles kaolinitiques consommées par géophagisme au Cameroun. Ann. Fac. Sci.; Univ. Yaoundé I, série Math-info-phys-chim, 31(2), 319-334.

Njoya, A. (2007). Etude du gisement de kaolin de Mayouom (Foumban, Ouest Cameroun): Géologie, Minéralogie et Géochimie (Doctor theory). Option géologie des matériaux, university Yaoundé I, $142+$ annexes.

Njoya, A., Nkoumbou, C., Grosbois, C., Njopwouo, D., Njoya, D., Courtin-Nomade, A., ... Martin, F. (2006). Genesis of Mayouom kaolin deposit (western Cameroon). Applied Clay Science, 32(1), 125-140. http://dx.doi.org/10.1016/j.clay.2005.11.005

Njoya, D. (2004). Minéralogie, propriétés physiques et mécaniques des céramiques des argiles de Mayouom (Cameroun) (Doctor theory). Option physico-chimie des matériaux minéraux, university Ydé. Cam. $89 \mathrm{p}+$ annexes.

Nyakairu, G. W., Koeberl, C., \& Kurzweil, H. (2001). The Buwambo kaolin deposit in central Uganda: Mineralogical and chemical composition. GEOCHEMICAL JOURNAL-JAPAN-, 35(4), 245-256. http://dx.doi.org/10.2343/geochemj.35.245

Ossah, N. H. (1975). Altération des roches volcaniques dans les Monts Bamenda, Cameroun. Géologie, minéralogie et géochimie (Doctoral dissertation). Thèse Doctorat 3e cycle, Université de Paris VI, France.

Petit, S. (1994). Hétérogénéité et variabilité de la composition chimique des minéraux argileux: à quelle échelle? (Discussion de la notion de solution solide, HDR, University Poitiers, France).

Pialy, P., Nkoumbou, C., Villieras, F., Njopwouo, D., Yvon, J., \& Bonnet, J. P. (2009). Characterization for ceramic applications of clay materials from Lembo deposit, Mount Bana (Cameroon). Silicates industriels, (11-12), 331-338.

Pialy, P., Nkoumbou, C., Villiéras, F., Razafitianamaharavo, A., Barres, O., Pelletier, M., ... \& Yvon, J. (2008). Characterization for industrial applications of clays from Lembo deposit, Mount Bana (Cameroon). Clay Minerals, 43(3), 415-435. http://dx.doi.org/10.1180/claymin.2008.043.3.07

Pruett, R. J., \& Murray, H. H. (1991). Clay mineralogy, alteration history, and economic geology of the Whitemud Formation, southern Saskatchewan, Canada. Clays and Clay Minerals, 39(6), 586-596. http://dx.doi.org/10.1346/CCMN.1991.0390604

Türkmenoğlu, A. G., \& Yavuz-Işık, N. (2008). Mineralogy, chemistry and potential utilization of clays from coal deposits in the Kütahya province, Western Turkey. Applied Clay Science, 42(1), 63-73. http://dx.doi.org/10.1016/j.clay.2007.11.006

Vidal, P. (1998). Géochimie. Série géosciences. Dunod, Paris, p. 190.

Wouatong, A. (1998). Mineralogical study of weathering on the Bana Complex, western Part of Cameroon. JOURNAL OF SCIENCE-HIROSHIMA UNIVERSITY SERIES C, 11, 55-99.

Wouatong, G. A., Kitagawa, R., Takeno, S., Tchoua, F. M., \& Njopwouo, D. (1996). Morpholocal transformation of kaolin minerals from granite saprolite in the western part of Cameroon. Clay Science, 10, 67-81.

Wronkiewicz, D. J., \& Condie, K. C. (1987). Geochemistry of Archean shales from the Witwatersrand Supergroup, South Africa: source-area weathering and provenance. Geochimica et Cosmochimica Acta, 5l(9), 2401-2416. http://dx.doi.org/10.1016/0016-7037(87)90293-6

Yvon, J., Lietard, O., Cases, J. M., \& Delon, J. F. (1982). Minéralogie des argiles kaoliniques des Charentes. Bull. Minéral, 105, 431-437.

\section{Copyrights}

Copyright for this article is retained by the author(s), with first publication rights granted to the journal.

This is an open-access article distributed under the terms and conditions of the Creative Commons Attribution license (http://creativecommons.org/licenses/by/3.0/). 